\title{
Carfentanil, el opioide que los anestesistas deberíamos conocer
}

\author{
Carfentanil, the opioid that anesthetists should know \\ Nicole Bloch², Lucas Oberpaur², Francisca Elgueta'
}

\begin{abstract}
Carfentanil is a synthetic opioid derived from fentanyl. It is a $\mu$-opioid receptor agonist with a potency 10,000 times greater than morphine and 30 times greater than fentanyl. It's currently only used on animals or in small doses for research purposes, but its use as an illicit drug has been increasing in the world in recent years. Little is known about its pharmacokinetics and pharmacodynamics in humans, most of the information being extrapolated from animal studies. The drug's high potency could be explained by its high lipophilicity, large distribution volume and potential active metabolites. It's a potent analgesic and sedative, with a large therapeutic index, but small lethal doses. Carfentanil poisoning is similar to any opioid toxidrome, the most common symptoms being loss of consciousness and respiratory depression. Like the rest of opioids, its antidote is naloxone, but multiple doses may be necessary for treating intoxications.
\end{abstract}

\section{RESUMEN}

El carfentanil es un opioide sintético derivado del fentanilo. Es un agonista del receptor opioide $\mu$ con una potencia 10.000 veces mayor a la morfina y 30 veces mayor al fentanilo. Actualmente su uso se limita a animales o, en dosis pequeñas, para investigación, pero su uso como droga ilícita ha ido en aumento en el mundo en los últimos años. Poco es lo que se conoce respecto a su farmacocinética y farmacodinamia en seres humanos, siendo estas extrapoladas de estudios en animales. Su alta potencia pudiera ser explicada por su alta lipofilicidad, gran volumen de distribución y por tener metabolitos activos. Es un analgésico y sedante potente, con un índice terapéutico grande, pero dosis letales pequeñas. El cuadro de intoxicación por carfentanil es similar a cualquier síndrome tóxico por opioides, siendo los síntomas más frecuentes el compromiso de conciencia y la depresión respiratoria. Su antídoto es la naloxona, al igual que el resto de los opioides, pero pudiendo ser necesario el uso de múltiples dosis para tratar intoxicaciones.

\section{Key words:}

Carfentanil, opioid, pharmacokinetics, pharmacodynamics

\author{
Palabras clave: \\ Carfentanyl, \\ opioides, \\ farmacocinética, \\ farmacodinámica
}

División de Anestesiología, Escuela de Medicina, Facultad de Medicina, Pontificia Universidad Católica de Chile. Alumno de Medicina, Pontificia Universidad Católica de Chile. Santiago, Chile.

Fecha de recepción: mayo de 2018

Fecha de aceptación: julio de 2018

\section{ORCID}

https://orcid.org/0000-0001-8349-8921

Correspondencia:

Dra. Francisca Elgueta

Email: panchielgueta@gmail.com 


\section{Introducción}

I carfentanil es un opioide sintético derivado del fentanilo, su potencia es 10.000 veces mayor a la morfina y 30 veces mayor al fentanilo[1]. Fue sintetizado por primera vez por laboratorios Janssen Pharmaceutical en 1974 bajo el nombre Wildnil y en 1984 fue aprobado como un tranquilizante intramuscular de grandes animales. Solo se encuentra disponible en ampollas de $10 \mathrm{ml}$ con una concentración de $3 \mathrm{mg} / \mathrm{mL}[1]$. Existe un escaso conocimiento respecto al fármaco, conociendo principalmente su farmacocinética y farmacodinámica por estudios en animales.

Dado que es un agonista opioide de alta afinidad, el [11C]-carfentanil radiomarcado es utilizado en el área de investigación para localizar receptores $\mu$ de opioides en humanos por tomografía por emisión de positrones (PET) con dosis entre 4,6 a 6,9 ug[2]. El uso en investigación es el único uso aprobado en humanos.

Es fundamental conocer en carfentanil, dado que su uso y abuso junto con otros análogos del fentanilo ha ido en aumento a lo largo de los años, convirtiéndose en un problema de salud pública en países del hemisferio norte[3]. De las 47.055 muertes por sobredosis de drogas que ocurrieron en Estados Unidos (EE. UU.) el año 2014, 28.647 (60,9\%) involucraron un opioide[4]. El carfentanil fue reconocido por primera como un aditivo a otras drogas de abuso a mediados de 2016 en EE.UU. y en Canadá donde fue reportada la intoxicación y convulsiones causadas por el carfentanil[5],[6].

A inicios de 2018 fue retirado su uso en animales por la FDA por solicitud del productor, Wildlife Laboratories, por su baja comercialización y su potencial abuso[7].

\section{Farmacología}

\section{Estructura molecular}

El carfentanil (IUPAC: Methyl 1-(2-phenylethyl)-4[phenyl(propanoyl)amino] piperidine-4-carboxylate) está compuesto por un grupo carboximetilo en la cuarta posición del anillo de piperidina del fentani$\mathrm{lo}[8]$.

\section{Presentaciones y vía de administración}

El carfentanil posee distintas presentaciones, como citrato se presenta como una solución clara, sin olor distintivo y altamente hidrosoluble[9] Como base libre existe como un polvo granular o cristalino blan$\mathrm{co}[1]$.
Se pueden utilizar distintas vías de administración. En animales las vías aprobadas son la oral, transmucosa, intramuscular e intravenosa[1].

En humanos se han identificado distintas formulaciones considerando que la principal exposición a carfentanil ocurre al consumir heroína o fentanilo adulterado[1]. Se ha identificado en polvo, tabletas, cápsulas, líquido y en papel secante[10]. Puede ser administrado por distintas vías, incluyendo la vía oral, subcutánea, intranasal, intravenosa e intramuscular. Además, dada su alta potencia, puede ser absorbido a través de la piel o inhalado[8].

\section{Farmacocinética}

Dado que la experiencia con carfentanil se limita a uso veterinario y estudios in vitro, existe escaso conocimiento sobre la farmacocinética del compuesto en humanos[1].

Un estudio in vitro realizado en microsomas hepáticos humanos demostró la degradación de carfentanil vía citocromo P 450 a 12 metabolitos, de los cuales todos fueron de fase I excepto un metabolito de fase Il y el glucurónido de uno de los metabolitos de fase I. Los principales fueron el norcarfentanil, metabolito común al fentanilo y otros de sus derivados, y M8, mediante la hidroxilación del anillo de piperidina, un metabolito exclusivo de este derivado del fentanilo. La vida media de eliminación en microsomas humanos fue de 7,8 minutos, en cambio en hepatocitos humanos el metabolismo fue mucho más lento, sin lograr reducir la cantidad de sustrato, sugiriendo que el factor limitante para la eliminación del carfentanil no sería el clearance metabólico[11].

La vida media de eliminación del carfentanil en humanos se ha reportado en dos estudios. En un estudio que incluyó 13 pacientes sanos con un seguimiento de 90 minutos tras la administración endovenosa de carfentanil, determinó una vida media de eliminación de 41,8 minutos (DS 17,5 min)[12]. En un reporte de caso de intoxicación de un paciente por carfentanil inhalado, con un seguimiento de 52 horas, la vida media fue de 5,7 horas para carfentanil y de 11,8 horas para su metabolito norcarfentanil[3].

Distintos mecanismos podrían explicar la lenta eliminación del carfentanil. Por una parte, existe una probable alta unión a proteínas, inferida por su similitud estructural a fentanilo y sufentanilo, que son de $84,4 \%$ y $92,5 \%$ respectivamente[3]. Por otra parte, el carfentanil tiene una alta liposolubilidad, dada por un $\log P$ calculado entre 3,67 y 3,7[13]. Ambos factores podrían explicar la prolongada vida media del fármaco en comparación con la tasa de eliminación en microsomas. Además, su alta lipofilicidad, le permite 
una gran penetración de la barrera hematoencefálica, lo que contribuye a su alta potencia[8].

\section{Farmacodinamia}

El carfentanil es un agonista de alta potencia del receptor opioide $\mu$. Los receptores de opioides son parte de un sistema neuroquímico con rol en el dolor, estrés, convulsiones, desórdenes psiquiátricos y enfermedades neurodegenerativas. Dentro de sus efectos reconocidos se encuentra su efecto sedante y analgésico[2].

\section{Sedación}

El carfentanil actúa principalmente en el sistema nervioso central. Similar a otros agonistas del receptor $\mu$ de opioides, genera una depresión del sistema nervioso central, suprime el reflejo de la tos y causa miosis de la pupila. Además de producir mareos y sedación[8].

En veterinaria se utiliza como tranquilizante dada su alta potencia y la rápida instalación de los efectos sedantes[8]. Por ejemplo, en ratones, la exposición a carfentanil en aerosol produjo pérdida de conciencia en 1 minuto[14]. En cabras domésticas, una dosis intramuscular de $40 \mathrm{mg} / \mathrm{kg}$ hizo que estos animales se durmieran en $84 \pm 18$ segundos posterior a la dosis[15].

\section{Analgesia}

La farmacodinámica de su potencia analgésica fue estudiada en ratas. Su potencia analgésica es aproximadamente 10.000 veces la de la morfina, 4.000 veces la de la heroína y 20-30 veces la del fentanilo[16].

Mediante estudios de unión receptor-ligando, se determinaron las constantes de disociación $\mathrm{K}_{\mathrm{i}}$ para receptores opioides $\mu, \delta$ y $\kappa$ humanos. Esta constante refleja la afinidad de una proteína por su ligando, y un menor valor de la constante refleja una mayor afinidad. Para todos los receptores esta constante fue menor en carfentanil $(0,024 \mathrm{nM}, 3,3 \mathrm{nM}$ y $43 \mathrm{nM}$, respectivamente) que en fentanilo $(1,9 \mathrm{nM}, 153 \mathrm{nM}$ y $197 \mathrm{nM}$, respectivamente). Esto sugiere una mayor afinidad del carfentanil por dichos receptores que el fentanilo[17].

\section{Índice terapéutico}

Se define índice terapéutico a la relación que compara la concentración plasmática de la droga en la que tiene un efecto terapéutico con la concentración en la que causa toxicidad en humanos o letalidad en animales. Puede ser calculada en animales como la dosis letal de la droga para el 50\% de la población (LD50) dividido por la dosis mínima efectiva para el
$50 \%$ de la población (ED50). Mientras mayor es el índice terapéutico, más segura es la droga[18].

Los valores que han sido reportados para el carfentanil provienen de estudios en ratas. Se describe un índice terapéutico de 10.600 en comparación con el del fentanilo que es de 300, además de un LD50 de $3,39 \mathrm{mg} / \mathrm{kg}$ [1]. El ED50 descrito es de 0,00032 mg/ $\mathrm{kg}[16]$.

\section{Efectos adversos}

Se han descrito algunos efectos adversos del uso de carfentanil en personas sanas. En un estudio imagenológico con PET, se le administró a personas sanas no consumidoras de drogas, una dosis de 0,019 $\mu \mathrm{g} /$ $\mathrm{kg}$ en bolo intravenoso. $60 \%$ de los sujetos reportaron efectos adversos, como mareos $(60 \%)$, náuseas (33\%), vómitos (7\%) y prurito (7\%)[12].

Tal como otros agonistas de receptores opioides, la intoxicación por carfentanil se manifiesta por un síndrome tóxico opioide, con compromiso de conciencia, depresión respiratoria y miosis, entre otros[1]. Dado que los estudios farmacodinámicos del carfentanil son en animales, se desconoce la dosis letal de carfentanil en humanos[8].

Se ha detectado carfentanil en múltiples casos de muertes por sobredosis, de manera aislada y también en combinación con otras drogas en EE.UU.[8].

El año 2002 en Rusia se utilizó un aerosol, posteriormente establecido que contenía carfentanil y remifentanil, en una toma de rehenes dentro de un teatro. Se introdujo por la ventilación 15 minutos previo a la entrada de las fuerzas especiales al recinto. De los 800 rehenes, 650 requirieron hospitalización y 127 fallecieron, con síntomas compatibles con intoxicación por opioides[1].

En 2010 se reportó un caso de intoxicación por parte de un veterinario que sufrió una salpicadura en la cara, ojos y boca con carfentanil tras un accidente con un dardo cargado con 1,5 mg del medicamento. Dentro de dos minutos, a pesar de descontaminarse con agua, presentó mareos y somnolencia por lo que se le administraron $100 \mathrm{mg}$ de naltrexona, logrando revertir la sintomatología[9].

\section{Antídoto}

Salvo los casos previamente mencionados, se desconoce la utilidad de antagonistas competitivos de receptores opioides para el tratamiento de la intoxicación con carfentanil en humanos. 
En un estudio realizado en uapitís sedados con 10 $\mathrm{mcg} / \mathrm{kg}$ de carfentanil, se logró mejoría de ésta en 5 minutos con una dosis de $20 \mathrm{mcg} / \mathrm{kg}$ de naloxona. Con ello se podría concluir que, a pesar de la alta potencia del carfentanil, no se requeriría de dosis mucho mayores a la habitual para revertir sus efectos[19]. Sin embargo, la dosis de naloxona efectiva debe ser determinada empíricamente con el objetivo de lograr una ventilación efectiva y pueden ser necesarias dosis repetidas[1]. En animales, en caso de no disponer de naloxona, se recomienda una dosis de naltrexona de $100 \mathrm{mg}$ por cada $1 \mathrm{mg}$ de carfentanil, sin embargo, se han reportado fenómenos de renarcotización, requiriendo dosis múltiples para lograr una reversión efectiva[1].

\section{Conclusión}

El carfentanil es un potente opioide que recientemente fue retirado del listado de medicamentos aprobados por la FDA para su uso en animales, por el riesgo potencial de uso inadecuado. Aún así es importante saber que, en buenas manos y con un uso racional, puede resultar un medicamento atractivo y un buen recurso farmacológico. Por otro lado, dado el aumento reciente de consumo recreacional de opioides sintéticos y por consiguiente intoxicaciones asociadas a carfentanil, conocer las propiedades farmacocinéticas y farmacodinámicas del carfentanil es crucial para médicos anestesistas para poder sospechar y tratar oportunamente a estos pacientes.

\section{Referencias}

1. Leen JL, Juurlink DN. Carfentanil: a narrative review of its pharmacology and public health concerns. Can J Anaesth. 2019 Apr;66(4):414-421. Disponible en: https://link.springer.com/ article/10.1007\%2Fs12630-01901294-y

2. Frost JJ, Douglass KH, Mayberg HS, Dannals RF, Links JM, Wilson $A A$, et al. Multicompartmental analysis of [11C]-carfentanil binding to opiate receptors in humans measured by positron emission tomography. J Cereb Blood Flow Metab. 1989 Jun;9(3):398-409. Disponible en: https://journals.sagepub.com/ doi/abs/10.1038/jcbfm.1989.59

3. Uddayasankar U, Lee C, Oleschuk C, Eschun G, Ariano RE. The Pharmacokinetics and Pharmacodynamics of Carfentanil After Recreational Exposure: A Case Report. Pharmacotherapy. 2018 Jun;38(6):e41-e45. Disponible en: https://accpjournals. onlinelibrary.wiley.com/doi/ full/10.1002/phar.2117 https:// doi.org/10.1002/phar.2117.

4. Rudd RA, Seth P, David F, Scholl L. Increases in Drug and Opioid-
Involved Overdose Deaths - United States, 2010-2015. MMWR Morb Mortal Wkly Rep. 2016 Dec 30;65(50-51):1445-1452. Disponible en: https://www. cdc.gov/mmwr/volumes/65/wr/ mm655051e1.htm

5. Rotuno-Johnson M. Ohio has most carfentanil seizures in United States. Associated Press, November 3, 2016. Disponible en: http://nbc4i.com/2016/11/03/ ohio-has-most-carfentanilseziures-in-united-states/

6. Special Advisory Committee on the Epidemic of Opioid Overdoses. National report: Apparent opioid-related deaths in Canada (January 2016 to June 2017) Web-based Report. Webbased Report. Ottawa: Public Heal Agency Canada; 2017. Disponible en: https://www.canada.ca/ en/public-health/services/publications/healthy-living/apparentopioid-related-deathsreport2016-2017-december.html

7. US Food and Drug Administration (FDA). The Opioid Epidemic: What Veterinarians Need to Know. 20 Aug. 2018. Disponible en: https://www.fda.gov/animalveterinary/resources-you/opioidepidemic-what-veterinarians- need-know

8. World Health Organization. Carfentanil: Critical Review Report. Agenda Item 4.8. Expert Comm Drug Depend 39th Meet. 2017; (November): 16-20. Disponible en: https://www.who. int/medicines/access/controlledsubstances/ecdd_39_meeting/ en/

9. George AV, Lu JJ, Pisano MV, Metz J, Erickson TB. Carfentanil--an ultra potent opioid. Am J Emerg Med. 2010 May;28(4):530-2. Disponible en: https://www.sciencedirect.com/science/article/ ii/5675710001178?via\%3Dihub

10. Drug Enforcement Agency (DEA). (2017). Fentanyl. A Briefing Guide for First Responders. Disponible en: https:// www.nvfc.org/wp-content/ uploads/2018/03/Fentanyl-Briefing-Guide-for-First-Responders. pdf

11. Feasel MG, Wohlfarth A, Nilles JM, Pang S, Kristovich RL, Huestis MA. Metabolism of Carfentanil, an Ultra-Potent Opioid, in Human Liver Microsomes and Human Hepatocytes by HighResolution Mass Spectrometry. AAPS J. 2016 Nov; 18(6):1489- 
1499. Epub 2016 Aug 5.

Disponible en: https://link. springer.com/article/10.1208\%2 Fs12248-016-9963-5

12. Minkowski CP, Epstein D, Frost JJ, Gorelick DA. Differential response to IV carfentanil in chronic cocaine users and healthy controls. Addict Biol. 2012 Jan;17(1):149-55. Disponible en: https://onlinelibrary. wiley.com/doi/full/10.1111/ j.1369-1600.2010.00256.x https://doi.org/10.1111/j.13691600.2010.00256.x.

13. Drugbank. Carfentanyl. $16 \mathrm{Dec}$. 2018. Disponible en: https:// www.drugbank.ca/drugs/ DB01535

14. Wong B, Perkins MW, Tressler J, Rodriguez A, Devorak J, Sciuto AM. (2017). Effects of inhaled aerosolized carfentanil on real-time physiological responses in mice: a preliminary evaluation of naloxone. Inhalation Toxicology, 29(2), 65-74. Disponible en: https://www. tandfonline.com/doi/full/10.1 080/08958378.2017.128206 5D80/08958378.2017.1282065 https://doi.org/10.1080/089583 78.2017.1282065.

15. Mutlow A, Isaza R, Carpenter JW, Koch DE, Hunter RP. (2004). Pharmacokinetics of carfentanil and naltrexone in domestic goats (Capra hircus). Journal of Zoo and Wildlife Medicine, 35(4), 489-496. Disponible en: https://bioone.org/journals/Journal-of-Zoo-and-Wildlife-Medicine/volume-35/issue-4/03-074/ PHARMACOKINETICS-OF-CARFENTANIL-AND-NALTREXONEIN-DOMESTIC-GOATS-spanclassgenus/10.1638/03-074. short

16. Van Bever WF, Niemegeers CJ, Schellekens $\mathrm{KH}$, Janssen PA. N-4-Substituted 1-(2-arylethyl)4-piperidinyl-N-phenylpropanamides, a novel series of extremely potent analgesics with unusually high safety margin. Arzneimittelforschung. 1976;26(8):1548-51. Disponible en: https://www.researchgate. net/publication/23120196_N4-Substituted_1-2arylethyl-4-piperidinyl-Nphenylpropanamides_a_no- vel_series_of_extremely_potent_analgesics_with_unusuaIly_high_safety_margin

17. Binding DB. The Binding Database - 2017. Disponible en: https://www.bindingdb.org/ jsp/dbsearch/PrimarySearch_ ki.jsp?energyterm=kJ/mole\&tag $=$ lidki\&monomerid=50012477\& column $=\mathrm{KI} \&$ startPg $=0$ \&Incremen $t=50$ \&submit=Seach

18. Tamargo J, Le Heuzey JY, Mabo P. Narrow therapeutic index drugs: a clinical pharmacological consideration to flecainide. Eur J Clin Pharmacol. 2015 May;71(5):549-67. Disponible en: https://link. springer.com/article/10.1007\%2 Fs00228-015-1832-0 https://doi. org/10.1007/s00228-015-18320 .

19. Cole JB, Nelson LS. Controversies and carfentanil: We have much to learn about the present state of opioid poisoning. Am J Emerg Med. 2017 Nov;35(11):1743-1745. Disponible en: https://www.sciencedirect.com/science/article/pii/0735 675717306940?via\%3Dihub 